\title{
Mechanical characteristics of microwave sintered silicon carbide
}

\author{
S MANDAL, A SEAL, S K DALUI, A K DEY, S GHATAK and A K MUKHOPADHYAY* \\ Central Glass and Ceramic Research Institute, Kolkata 700 032, India
}

\begin{abstract}
The present work deals with the sintering of $\mathrm{SiC}$ with a low melting additive by microwave technique. The mechanical characteristics of the products were compared with that of conventionally sintered products. The failure stress of the microwave sintered products, in biaxial flexure, was superior to that of the products made by conventional sintering route in ambient condition. In firing of products by conventionally sintered process, $\mathrm{SiC}$ grain gets oxidized producing $\mathrm{SiO}_{2}(\sim 32 \mathrm{wt} \%)$ and deteriorates the quality of the product substantially. Partially sintered silicon carbide by such a method is a useful material for a varieties of applications ranging from kiln furniture to membrane material.
\end{abstract}

Keywords. Microwave sintering; biaxial flexure; silicon carbide.

\section{Introduction}

Silicon carbide $(\mathrm{SiC})$ ceramics is a very well known candidate material for a structural application. However, due to (i) poor densification due to highly directional bonding, (ii) susceptibility of $\mathrm{SiC}$ to $\mathrm{SiO}_{2}$ conversion in presence of air/oxygen and (iii) prohibitively high cost of finished product, its commercial success has been limited. Additives play a major role in the conventional sintering of $\mathrm{SiC}$ (Lee et al 1995, 1996), and, the application of microwave energy for sintering of such ceramics has recently become an attractive area of research and development. The major advantages of microwave processing of ceramic materials are manifold, viz. (i) accelerated densification rate as a result of direct, instantaneous, volumetric heating, (ii) controlled grain growth, (iii) uniform microstructure and (iv) achievement of high density without the usage of sintering aids (Lee and Case 1999; Goldstein et al 1999). Several studies have reported on the effect of using microwave method of sintering ZTA (Lee and Case 1999), Y-PSZ (Goldstein et al 1999), $\mathrm{MgAl}_{2} \mathrm{O}_{4}$ (Goldstein 1998), alumina (Xie et al 1999), joining of ceramic composites (Aravindan and Krishnamurthy 1990), SiC/alumina cement composites (Leiser and Clarke 1998) etc.

The purpose of the present work was to prepare $\mathrm{SiC}$ based porous, partially densified bodies for low value, high volume commercial applications, e.g. as kiln furniture and ceramic membranes for filtration. The samples were synthesized by the conventional as well as microwave heating process. Further, the mechanical properties like flexural strength, hardness and fracture toughness were compared for samples densified by both the above mentioned techniques.

*Author for correspondence

\section{Experimental}

The low melting sintering additives (10 $\mathrm{wt} \%)$ were mixed with $\alpha$-SiC powder (source: Grindwell Norton India Ltd., India, surface area $-1.09 \mathrm{~m}^{2} / \mathrm{g}$ ) thoroughly by the process reported elsewhere (Ghatak and Dhargupta 1995). After thorough mixing of the powder, bars and pellets were fabricated by isostatic pressing at $150 \mathrm{MPa}$ pressure.

For conventional sintering process bars and pellets were placed in a muffle furnace, and fired at $1350^{\circ} \mathrm{C}$ for a soaking time of about $15-45 \mathrm{~min}$ in ambient atmosphere. The thickness of the samples to be sintered in the microwave were varied from $1 \mathrm{~mm}$ to $5 \mathrm{~mm}$, to ascertain the maximum allowable thickness of the sample for such processing technique. For the microwave heating process, a specially designed applicator placed in a domestic microwave oven was used (Mukhopadhyay et al 2001). The oven had a output power level tunable up to a maximum of 800 watt and operating frequency of $2.45 \mathrm{GHz}$. The firing temperature was measured using a Pt/Pt-13\% $\mathrm{Rh}$ thermocouple. The accuracy of the measurement was $\pm 10^{\circ} \mathrm{C}$. The phase purity of the fired product was checked by the X-ray diffraction (XRD) technique with a $\mathrm{Cu}-\mathrm{K}_{\alpha}$ target. The density ( $\rho$ ) data of the fired samples $(45 \mathrm{~mm} \times 5 \mathrm{~mm} \times 3 \mathrm{~mm})$ were measured by Archimedes' principle.

The biaxial flexural strength was measured by the conventional ball-on-ring technique (de With and Wagemans 1989). The hardness data was obtained for an applied load of $1 \mathrm{~N}$ using a microhardness tester fitted with a Vicker's square pyramidal indenter. The fracture toughness data was obtained by SENB technique from bars of relative notch depth $(a / w)$ of $0.3-0.5$ under a three point loading system with a span of $40 \mathrm{~mm}$ and a crosshead speed of $1 \mathrm{~mm} / \mathrm{min}$ (Brown and Srawley 1966). Microstructure analysis of the fractured surface was conducted by the scanning electron microscope. 


\section{Results and discussion}

It is evident from the sintering data presented in table 1, that the percentage of weight change in products heated in microwave was insignificant. But an appreciable weight gain was observed for conventionally fired specimen.

With an increase in the thickness of the microwave fired specimen some decrease in the values of the linear shrinkage and bulk density were observed. This might be due to the limitation of the incident microwave energy penetration in these specimens.

The data on the biaxial flexural strength, hardness and fracture toughness $\left[K_{\mathrm{IC}}\right]$ values for both conventionally fired and microwave fired samples are presented in table 2. The biaxial fracture strength of the microwave fired samples, generally, were much higher than those of the conventionally sintered samples. However, the hardness data were not much altered though the fracture toughness of the conventionally fired product was slightly higher than that of the microwave fired specimen. Scanning electron micrograph revealed that there was some abnormal grain growth (figure 1) as well as very large cracks (figure 2) present in the samples tested for $K_{\mathrm{IC}}$ determination. A large grain may act as a critical flaw of large dimension and hence, may help in the lowering of the fracture toughness value.

Similarly, the presence of a large crack e.g. as in figure 2 , may cause local stress concentration and hence, cause a lowering of the fracture toughness value. But, in general the average microstructure of the microwave sintered

Table 1. Sintering data of the fired samples.

\begin{tabular}{lcccc}
\hline $\begin{array}{l}\text { Sample } \\
\text { no. }\end{array}$ & $\begin{array}{c}\text { Thickness } \\
(\mathrm{mm})\end{array}$ & $\% \Delta l$ & $\% \Delta w$ & $\begin{array}{c}\text { B.D. } \\
\text { (g/cc) }\end{array}$ \\
\hline M-1 & 1 & 4.20 & -0.98 & 1.965 \\
M-2 & 2 & 4.10 & -0.96 & 1.956 \\
M-3 & 3 & 4.08 & -0.92 & 1.950 \\
M-4 & 4 & 3.75 & 0.09 & 1.944 \\
M-5 & 5 & 3.50 & 0.035 & 1.915 \\
C-5 & 5 & 3.17 & 15.95 & 1.852 \\
\hline
\end{tabular}

$\mathrm{M}$, Microwave heating system; $\mathrm{C}$, conventional heating system; $\% \Delta w=$ per cent weight loss; $\% \Delta l=$ per cent linear shrinkage.

Table 2. Mechanical properties of fired samples.

\begin{tabular}{lcccc}
\hline $\begin{array}{l}\text { Sample } \\
\text { no. }\end{array}$ & $\begin{array}{c}\text { Thickness } \\
(\mathrm{mm})\end{array}$ & $\begin{array}{c}\text { Biaxial } \\
\text { strength } \\
(\sigma, \mathrm{MPa})\end{array}$ & $\begin{array}{c}\text { Hardness } \\
\left(\mathrm{kg} / \mathrm{mm}^{2}\right)\end{array}$ & $\begin{array}{c}K_{\mathrm{IC}} \\
\left(\mathrm{MPa} \cdot \mathrm{m}^{1 / 2}\right)\end{array}$ \\
\hline M-1 & 1 & $27 \cdot 006$ & $97 \cdot 2$ & - \\
M-2 & 2 & 26.362 & $96 \cdot 5$ & - \\
M-3 & 3 & $25 \cdot 223$ & $94 \cdot 4$ & - \\
M-4 & 4 & 21.041 & 89.7 & - \\
M-5 & 5 & $20 \cdot 167$ & $69 \cdot 0$ & $0 \cdot 4$ \\
C-5 & 5 & 14.847 & $95 \cdot 4$ & $0 \cdot 7$ \\
\hline
\end{tabular}

product was less porous (figure 3 ) than that of the conventionally sintered product (figure 4). Hence, a higher flexural strength was observed for the microwave sintered samples (M1 to M5). Even at a comparable thickness, the strength of the microwave fired product was twice as much as that of the conventionally fired product. Such a large difference may not solely be due to the difference in porosity distribution of the samples prepared by the two different firing techniques.

The appreciable weight gain in the conventionally fired product, could be due to oxidation of $\mathrm{SiC}$, e.g. $50 \%$ weight gain of a green $\mathrm{SiC}$ sample correspond to $100 \%$ conversion of the $\mathrm{SiC}$ to $\mathrm{SiO}_{2}$ according to the following equation

$$
\mathrm{SiC}+\mathrm{O}_{2}=\mathrm{SiO}_{2}+\mathrm{CO} / \mathrm{CO}_{2}
$$

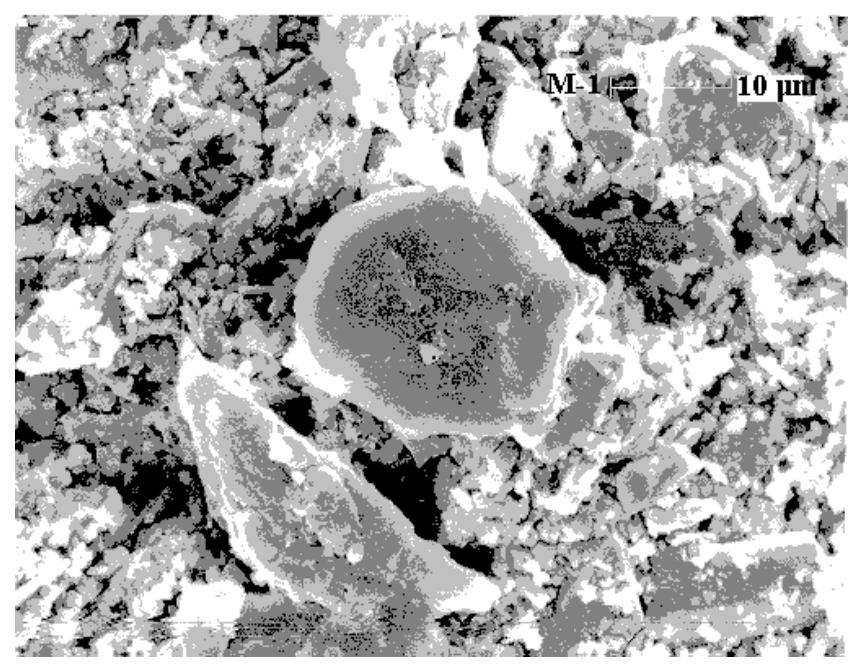

Figure 1. Microstructure of the fractured surface of the microwave fired sample showing agglomerated grains.

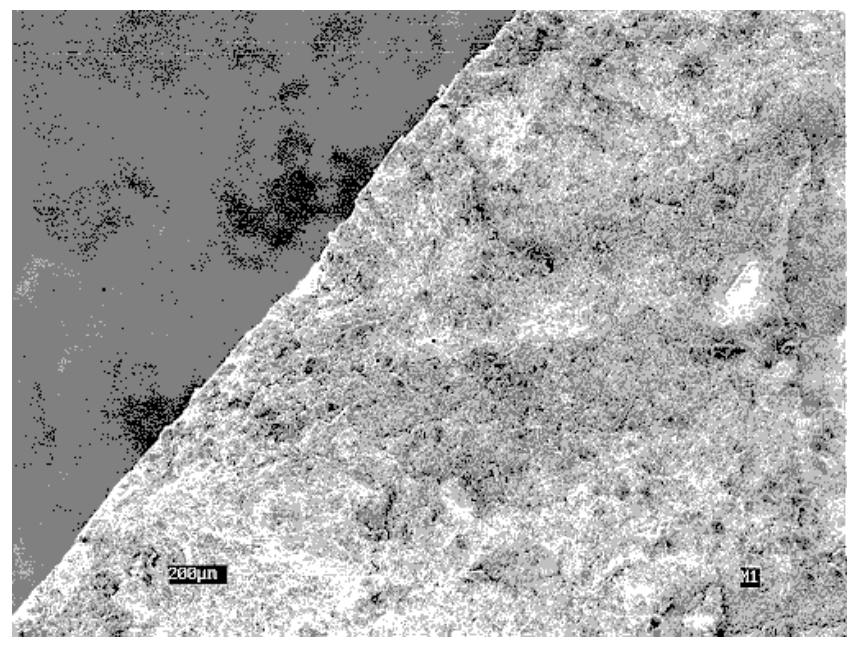

Figure 2. Microstructure of the fractured surface of the microwave fired sample showing a microcrack on the tensile surface. 
It was indicative therefore, that in the conventionally fired product about $32 \%$ conversion of $\mathrm{SiC}$ to $\mathrm{SiO}_{2}$ had occurred. However, the formation of $\mathrm{SiO}_{2}$ was negligible in the microwave heating process.

To appreciate this difference, it is imperative to look into the basic steps involved in the microwave heating process. For any given dielectric, ideally, the electric charge should adjust itself instantaneously to any change in applied voltage $V_{\mathrm{a}}$. Thus, the rate of heat generation in an ideal dielectric is zero because the product of the voltage and charging current disappears. This is so because, the charging current is $90^{\circ}$ advanced in phase, ideally, with respect to the applied voltage

$$
V_{\mathrm{a}}=V_{0} \sin 2 \pi f_{\mathrm{a}} t_{\mathrm{a}},
$$

where $f_{\mathrm{a}}$ stands for the frequency of the applied voltage of time period $t_{\mathrm{a}}$. In reality, however, there is an inertia to the charge movement, that shows up as a relaxation time, $t_{\tau}$. For all real dielectric materials, therefore, the rate of heat generation has some finite value depending on the finite response time. The extent of such a heat generation is linked also with the type and extent of polarization that might occur due to the very presence of the applied field. The four types of polarization contributing for a dielectric

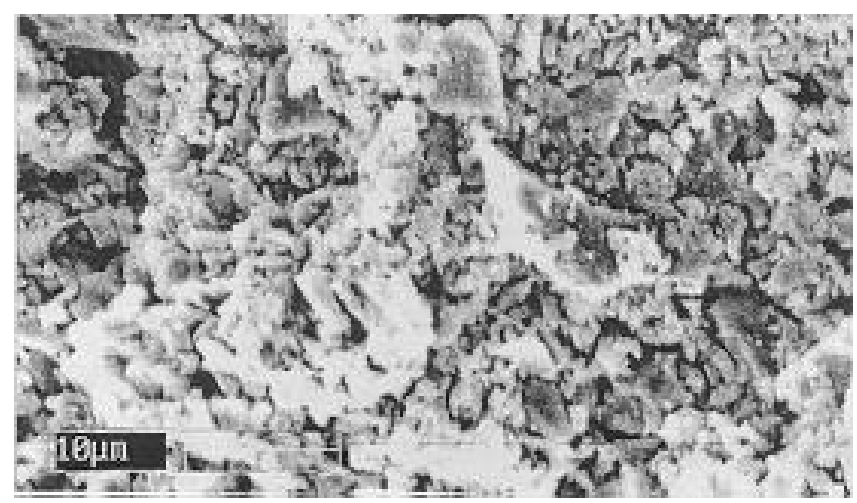

Figure 3. Microstructure of the fractured surface of the microwave fired sample.

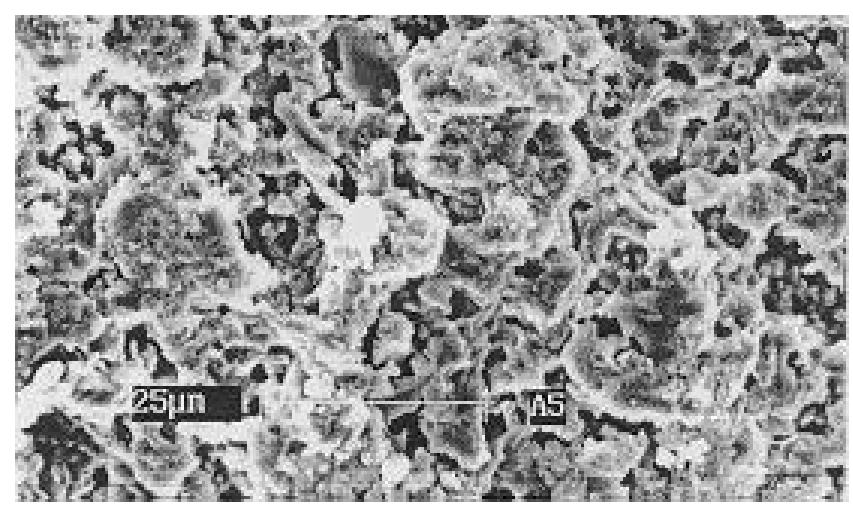

Figure 4. Microstructure of the fractured surface of the conventionally fired sample. material are electronic, ionic, orientational and spacecharge. The relative predominance of one type of polarization over another in a given system, however, is a detailed and complicated function of several factors such as the nature of bonding, the nature of majority charge carriers etc. This is exemplified by the following relationship where the power absorbed by the material and converted to heat per unit volume is

$$
P_{\mathrm{ab}}=\omega \cdot \varepsilon_{0} \cdot \varepsilon_{\mathrm{eff}}^{\prime \prime} \cdot E_{\mathrm{rms}}^{2}=\omega \cdot \varepsilon_{0} \cdot \varepsilon^{\prime} \cdot \tan \delta_{\mathrm{eff}} \cdot E_{\mathrm{rms}}^{2}
$$

where, $\omega=2 \pi f$ and $f$ is the frequency, $\varepsilon_{\mathrm{eff}}^{\prime \prime}$ the effective loss factor that includes all loss mechanisms and $E_{\mathrm{rms}}$ the root mean square of the electric field inside the material. The comparison of XRD data between the microwave and the conventionally sintered samples is shown in figures 5 and 6 . The crystoballite peak in the conventionally sintered $\mathrm{SiC}$ was more prominent.

In the case of microwave sintered $\mathrm{SiC}$, the $\mathrm{SiC}$ being predominantly covalent material $(\approx 84 \%$ covalency), and the operating microwave frequency is $2.45 \mathrm{GHz}$, the possibility of ionic polarization, space charge and electronic polarization can be overruled. Hence, only the orientational polarization mechanism might govern the polarization process.

It is evident from the aforesaid discussion that the volumetric heating occurred in microwave oven during sintering of $\mathrm{SiC}$ samples. Moreover, the rapid heating facilitated the prevention of the oxidation of $\mathrm{SiC}$ as evidenced by the presence of a small cristobalite peak in the XRD data of microwave heated SiC compact (figure 5). Conversely, in a conventional heating process, consolidation by sintering is initiated from the surface and gradually propagates to the interior of the sample. This makes the silicon carbide grain at the surface vulnerable to oxidation and hence, the presence of large amount of cristobalite formation, as revealed from the XRD data (figure 6).

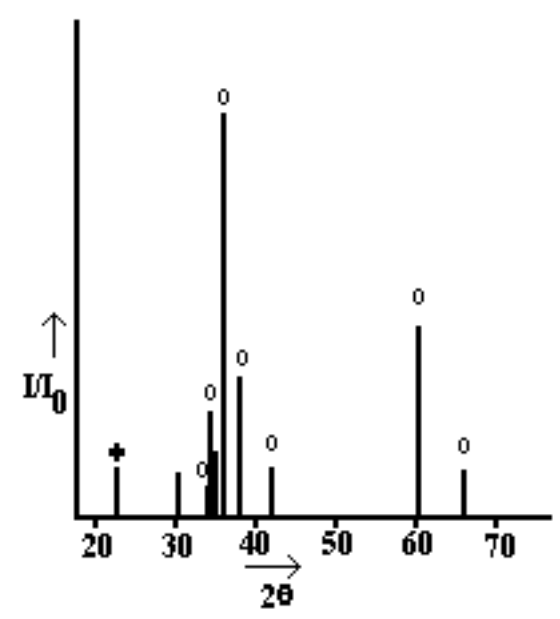

Figure 5. XRD lines of microwave fired silicon carbide. 


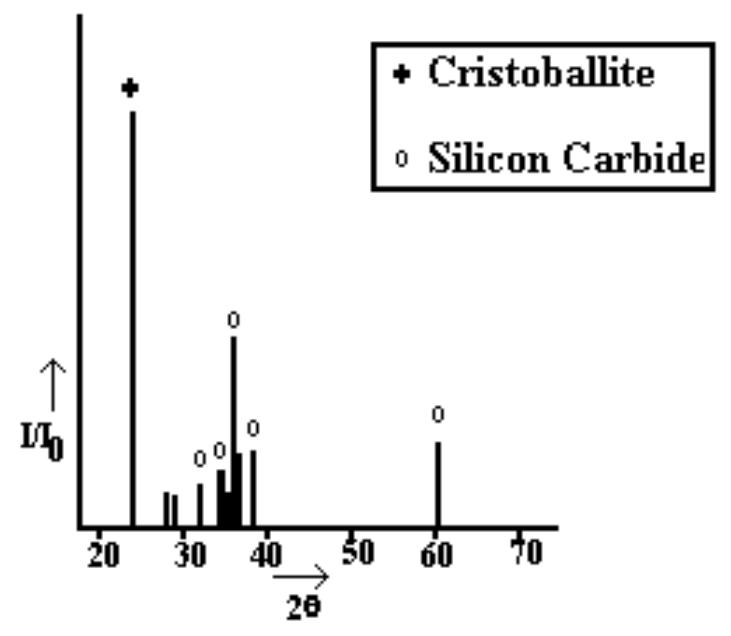

Figure 6. XRD lines of conventionally fired silicon carbide.

The formation of cristobalite having $\rho=2.33 \mathrm{~g} / \mathrm{cc}$ against $\mathrm{SiC}$ with $\rho=3.21 \mathrm{~g} / \mathrm{cc}$ resulted in the increase of the volume of the specimen and the formation of cristobalite having also a different coefficient of thermal expansion value to that of silicon carbide would create high internal strain within the specimen. This could be the reason why poor biaxial flexural strength is observed for the conventionally sintered specimen as compared to the microwave sintered sample.

\section{Conclusions}

The major conclusions of the present work are:
(I) Microwave heating process leads to a very low cristobalite formation as compared to the conventional sintering process.

(II) The biaxial flexural strength of the microwave heated SiC compact was higher than that of the conventionally heated SiC compact. However, the $K_{\mathrm{IC}}$ data of the former were slightly lower than that of the latter.

\section{References}

Aravindan S and Krishnamurthy R 1990 Mater. Lett. 38 245

de With G and Wagemans H H M 1989 J. Am. Ceram. Soc. 72 1538

Evans A G and Langdon T G 1976 Mater. Sci. 21171

Ghatak S and Dhargupta K K 1995 Patent No 1099, India

Goldstein A, Giefman L and Bar Ziv S 1998 J. Mater. Sci. Lett. 17977

Goldstein A, Travitzky N, Singurindy A and Kravchik M 1999 J. Eur. Ceram. Soc. 192067

Lee K Y and Case E D 1999 J. Mater. Sci. Lett. 18201

Lee J K, Tanaka H and Otani S 1995 J. Ceram. Soc. Japan 103 873

Lee J K, Tanaka H and Rim H 1996 Mater. Lett. 291

Leiser K S and Clarke D B 1998 Ceram. Engg. Sci. Proc. 19 367

Mukhopadhyay A K, Ray Chowdhury M, Seal A, Dalui S K, Banerjee M and Phani K K 2001 Bull. Mater. Sci. 24125

Xie Z, Yang J, Huang X and Huang Y 1999 J. Eur. Ceram. Soc. 19381 